\title{
Hypotension Investigation, Prospective Clinical Study
}

\author{
Ian Piper ${ }^{1}$, Alfonsas Vainoras ${ }^{2,3}$, Kristina Berskiene ${ }^{2,3}$, Rimtautas Ruseckas ${ }^{2,4}$, Vidmantas Jurkonis ${ }^{2,4}$, \\ Liepa Bikulciene ${ }^{2}$, Zenonas Navickas ${ }^{2}$, Dovile Karaliene ${ }^{2}$, Arminas Ragauskas ${ }^{2}$, \\ Mantas Deimantavicius ${ }^{2}$ \\ ${ }^{1}$ Clinical Physics, Southern General Hospital, \\ Glasgow, Scotland, UK \\ ${ }^{2}$ Health Telematics Science Institute, Kaunas University of Technology, \\ Barsausko St. 59 A553-A561, LT-51423 Kaunas, Lithuania \\ ${ }^{3}$ Sports Institute, Medical Academy, Lithuanian University of Health Sciences \\ Tilzes St. 18, LT-47181 Kaunas, Lithuania \\ ${ }^{4}$ Institute of Cardiology, Medical Academy, Lithuanian University of Health Sciences \\ Sukileliu av., LT-50140 Kaunas, Lithuania \\ vytautas.petkus@ktu.lt
}

\begin{abstract}
Hypotension occurring in the initial phase of resuscitation is significantly associated with increased mortality following brain injury, even when episodes are relatively short. A large amount of data exists in health care systems providing information on the major health indicators of patients in hospitals. It is believed that if enough of these data could be drawn together and analysed in a systematic way, then a system could be built that will trigger an alarm predicting the onset of a hypotensive event. In the paper the mathematical information algorithm based on the concept of the rank of a sequence is presented. For the analysis of hypotension physiology an application of a new algebraic method is proposed for real world time series analysis. Numerical experiments with a hypotension crisis prevention using arterial blood pressure time series are used to illustrate the potential of the proposed method. The algorithm for finding ranks of a sequence of the ECG parameters is presented in the paper in order to show complexity profiles. Experimental results show that presented in this paper method also can be used together with other hypotension prediction methods.
\end{abstract}

Index Terms-Hypotension physiology; time series; Hankel atrices; rank; complexity profile.

\section{INTRODUCTION}

Hypotension occurring in the initial phase of resuscitation is significantly associated with increased mortality following brain injury, even when episodes are relatively short. These prospective data reinforce the need for early continuous monitoring and improved treatment of hypotension in braininjured patients. The ability to predict adverse hypotensive events, where a patient's arterial blood pressure drops to abnormally low (and dangerous) levels, would be of major benefit to the fields of primary and secondary health care, and especially to the traumatic brain injury domain.

A large amount of data exists in health care systems

Manuscript received 12 May, 2015; accepted 22 December, 2015.

This research work has been funded by project „R\&D of innovative system for prognosis of arterial hypotension events", Ministry of Education and Science Lithuania. Grant number: VP1-3.1-ŠMM-10-V-02-003. providing information on the major health indicators of patients in hospitals (blood pressure, temperature, heart rate, etc.). It is believed that if enough of these data could be drawn together and analysed in a systematic way, then a system could be built that will trigger an alarm predicting the onset of a hypotensive event.

This was the basis for the Avert-IT project (http://www.avert-it.org), a collaborative EU-funded project involving the construction of a hypotension alarm system exploiting Bayesian neural networks using techniques of data federation to bring together the relevant information for study and system development. It must be noticed that before this project wasn't solution to this hypotensive prediction problem on the market and the commercialization aspects of the project were important. A variety of commercial systems do allow the prediction of forthcoming clinical states, but none are able to provide any probabilistic measure of the causative information that can be tied to context. For example, the Bio Sign device [Pulsewave ${ }^{\mathrm{TM}}$ devices: http://www.biosign.com/pulsewave-max.aspx] produces an index predicting cardiovascular instability based on several vital signs such as heart rate, respiration rate, etc.

The main methods for hypotensive prediction problem are listed below. The Genetic Algorithms (GA) providing a search technique that involves generating a random selection of solutions across the domain, then testing each of them against a fitness function. Though useful in scheduling and timetabling optimizations, there is some doubt as to whether the GA would be applicable in this system. The Bayesian Belief Networks (BBN) offering a probabilistic model that represents a set of variables and their probabilistic dependencies. Inherently capturing probability in its development, training $\mathrm{BBN}$ architecture with data-driven mechanisms has proven difficult. Artificial Neural Networks (ANN) - comprise a computer-simulated approach derived from the brain model, where individual neurons are interconnected from an input layer, through one or more hidden layers, to an output layer. 
In the Avert-IT project regarding to the various advantages and disadvantages of the different approaches, it has been agreed that a Bayesian approach to training an Artificial Neural Network (a BANN) is an effective way of training the system to detect the onset of a hypotensive event based on the input data from associated parameters [1]. The motivation for considering the BANN was its efficiency for the classification and modelling of highly nonlinear relationships whilst also considering probabilistic factors, which themselves are expected to be a major aspect in the clinical inputs involved.

The last years' studies showed that the complexity in human body functioning is important area of research. Measuring the complexity of various bio signals, such as ECG, EEG or DNA is a common task in medicine. The complexity of ECG signals is an important characterization of a process and might be used as a diagnostic tool.

Time series prediction is a challenging problem in many fields of science and engineering as well as have application in medicine and forecasting procedures include different techniques and models.

In general, the objective of these techniques is to build a model of the process and then use this model on the last values of the time series to extrapolate past behaviour into future.

A number of various mathematical methods, algorithms and computerized ECG analysis systems have been proposed for the analysis of ECG complexity, for example, entropybased algorithms [2]-[4], spectral analysis [5], chaos-based algorithms [6], [7], hidden Markov chains [8], Lempel-Ziv method [9] and other methods. Most of these analysis algorithms are heuristic and evaluate global features of processes and are not able to detect local features of dynamical processes.

Here is presented the mathematical information algorithm based on the concept of the rank of a sequence [10].

The concept of the rank of a sequence have been successfully used to express solutions of nonlinear differential equations in forms comprising ratios of finite sums of standard functions [11]-[13], for time series forecasting [14], logistic-matrix representation, research of chaos and for ECG complexity analysis [15].

The task of the presented algorithm is to develop strategy for finding the ranks of sequences of the ECG parameters.

\section{INVESTIGATED CONTINGENT}

In study where investigated 42 persons from UK, Glasgow Institute of Neurological Sciences at Southern General Hospital. Where studied 13 women, age (mean \pm SD) $58.85 \pm 13.02$ and $29 \mathrm{man}$, age 46,28 $\pm 17,27.26$ patients where with brain injury and 16 with other diseases may influence on arterial blood pressure (ABP).

All patients where monitored in coronary care unit and with expected hypotension reactions. We have used the Edinburgh University Secondary Insult Grades (EUSIG) definitions for hypotension (systolic arterial pressure $<90$ $\mathrm{mmHg}$ or mean arterial pressure $<70 \mathrm{mmHg}$ ).

\section{REGISTERED DATA}

At first from waveform signals data file the ECG and pressure signals are collected and in every RR interval (hearth rate) of ECG the systolic and diastolic blood pressure parameters are calculated finding global maximum and minimum of waveform signal in every cardio cycle (Fig. 1) as well as reducing noise level using standard filters, Fig. 2 .

In Fig. 2 the systolic and diastolic blood pressures values by black and grey lines accordingly and noise by dotted line are presented.

Synchronously to ABP was recorded ECG II standard lead. For every cardio cycle of ECG where measured - RR interval, time duration between two consecutive $\mathrm{R}$ waves of the ECG, JT interval, from the junction of J-point to T-wave end of the ECG and QRS complex from the beginning of Qwave to J-point of the ECG. For final analysis where used three synchronised data time sequences - RR, JT and QRS (Fig. 3).

\section{Methodology of AnAlysis}

General sequence of analysis procedures is presented in Fig. 3. All data, ABP and ECG where recorded and measured synchronously.

Hankel matrix analysis. Let us consider a sequence: $p_{0}, p_{1}, \ldots:=\left(p_{j} ; j \in Z_{0}\right)$ where elements $p_{j}$ can be real or complex numbers. Then, a sequence of Hankel matrixes reads

$$
H_{n}:=\left(p_{i+j-2}\right)_{1 \leq i, j \leq n}=\left[\begin{array}{cccc}
p_{0} & p_{1} & \cdots & p_{n-1} \\
p_{1} & p_{2} & \cdots & p_{n} \\
& & \cdots & \\
p_{n-1} & p_{n} & \cdots & p_{2 n-2}
\end{array}\right],
$$

where $n=1,2, \ldots$.

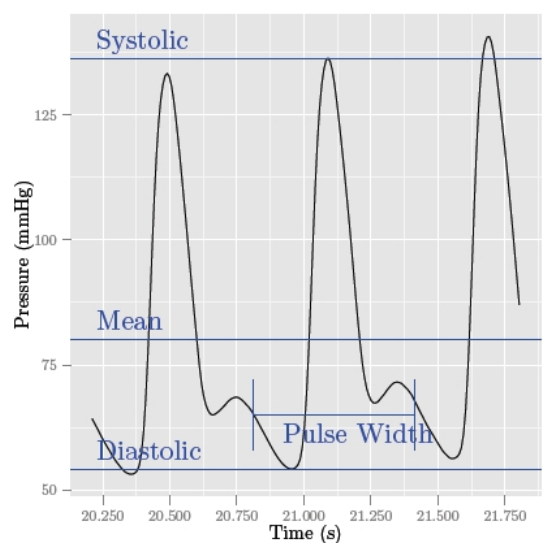

Fig. 1. Arterial blood pressure pulse with the main clinical concepts: pulse width, diastolic, mean and systolic highlighted.

The Hankel transform (the sequence of determinants of Hankel matrixes $)\left(d_{n} ; n \in N\right)$ reads

$$
d_{n}:=\operatorname{det} H_{n}
$$




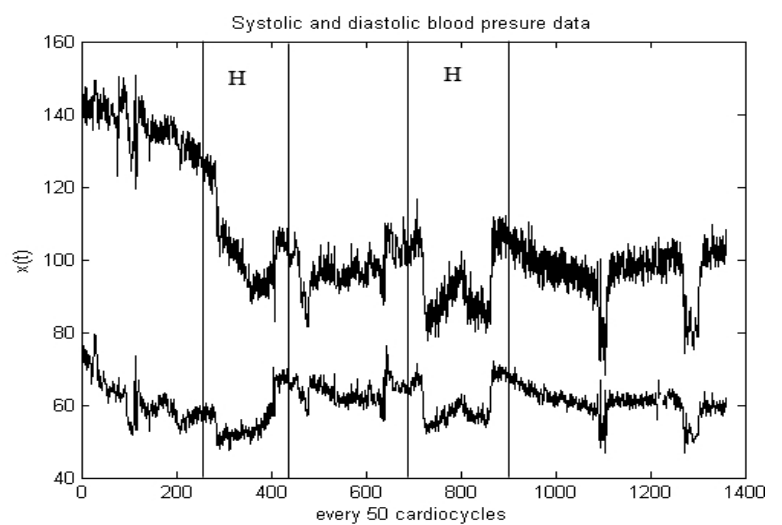

Fig. 2. Systolic and diastolic blood pressure data with hypotension (every 50 cardio cycles for representation clarity).

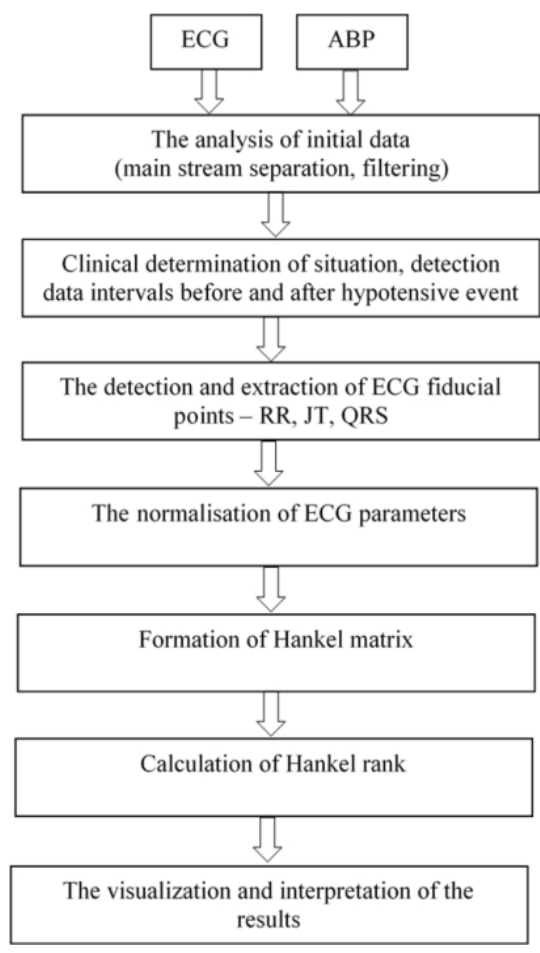

Fig. 3. General sequence of analysis procedures.

Definition 1. The sequence $\left(p_{j} ; j \in Z_{0}\right)$ has a rank $m \in Z_{0} ; m<+\infty$

$$
\operatorname{rank}\left(p_{j} ; j \in Z_{0}\right)=m
$$

if the sequence of determinants of Hankel matrixes has the following structure

$$
\left(d_{1}, d_{2}, \ldots, d_{m}, 0,0, \ldots\right)
$$

where $d_{m} \neq 0$ and $d_{m+1}=d_{m+2}=\ldots=0$.

Example 1. Let $p_{j}:=j^{2}, \quad j \in Z_{0}$. Then, $\operatorname{rank}\left(j^{2} ; j \in Z_{0}\right)=3$ because the sequence of determinants of Hankel matrices reads $(0,-1,-8,0,0, \ldots)$.

In computer-based realization it was considered that determinant of Hankel matrix equals zero, if its' value is lesser than the fixed precision $\varepsilon$.

In Table I average ranks for ECG parameters before hypotension were presented.
TABLE. I. HANKEL MATRIX RANKS (H) CALCULATED FOR INVESTIGATED PERSONS, FOR RR, JT AND QRS PARAMETERS BEFORE ABP DROPS DOWN AND DURING LOWERING OF ABP. $\mathrm{H}(\mathrm{RR}) 1$ IS BEFORE ABP FALL AND H(RR) 2 IS DURING ABP FALL, THE SAME FOR JT AND QRS PARAMETERS.

\begin{tabular}{|c|c|c|c|c|c|c|}
\hline Parameter & $\begin{array}{c}\text { H(RR) } \\
\mathbf{1}\end{array}$ & $\begin{array}{c}\text { H(RR) } \\
\mathbf{2}\end{array}$ & $\begin{array}{c}\text { H(JT) } \\
\mathbf{1}\end{array}$ & $\begin{array}{c}\text { H(JT) } \\
\mathbf{2}\end{array}$ & $\begin{array}{c}\text { H(QRS) } \\
\mathbf{1}\end{array}$ & H(QRS) 2 \\
\hline $\begin{array}{c}\text { Average H, } \\
\mathrm{N}=42\end{array}$ & 3.47 & 3.45 & 3.87 & 3.83 & 4.10 & 3.99 \\
\hline STDEV & 0.674 & 0.657 & 0.436 & 0.530 & 0.771 & 0.818 \\
\hline SEM & 0.104 & 0.101 & 0.067 & 0.082 & 0.119 & 0.126 \\
\hline
\end{tabular}

Algorithm 1. Algorithm for finding ranks of a sequence of the ECG parameter:

Input: $p_{0}, p_{1}, p_{2}, p_{3}, \ldots, p_{N-1}$ (a sequence of the ECG parameter), $0<\varepsilon \leq 1, s:=0$;

Step 1. Assign $n:=1$;

Step 2. Construct $H_{n}$ (1) and compute $d_{n}$ (2);

Step 3. Check if $d_{n}<\varepsilon$; if true, then save $m_{s}:=n-1$, $s:=s+1$; and repeat step 1 with the sequence $p_{s}, p_{s+1}, p_{s+2}, \ldots, p_{N-1}$. If false, then $n:=n+1$ and repeat step 2 .

Output: $m_{0}, m_{1}, m_{2}, \ldots-$ array of ranks of a given sequence (Fig. 4)

\section{RESUlts}

For the analysis of ECG parameters complexity and its profile, we used understanding of processes interactions interpretation according Bar-Yam [16], named as a "complexity profile". In complex systems we can see different fractal levels, closely interacting. Looking into heart and human organism as a complex system with few fractal levels (organism-heart-heart structures) we can postulate, that different ECG parameters with different time scale, can represent different fractal levels of organism. Interval RR could be related with organism regulatory features, JT interval could be related with heart metabolic features and interval QRS could be related with heart itself regulatory features. Calculated Hankel matrix ranks are presented in Table I for two situations: 1 - episode before ABP drops down and 2 - during ABP drops down. Data in Table I shows, that complexity for different parameters significantly differs but for episode before ABP fall down and during such episode difference is very small, not significant (Table I).

Having possibility to measure of RR, JT and QRS intervals complexity with help of Hankel matrix rank, it's possible to represent different clinical situations with complexity profile, where the largest scale is RR interval, middle scale is JT interval and smallest scale is QRS duration. According Theory of complex systems [16] smaller scale has complexity higher or the same as bigger scale. With increasing of scale, complexity, usually, is going down. These situations we have in all our investigated groups. Complexity profile, one line connecting complexities $(\mathrm{H})$ of RR, JT and QRS in Fig. 4 are shown for two groups of persons - with hypotension episodes (Hy) and without. Comparison of complexity data for these two groups shows, 
that persons who have real situation of hypotension, have smaller complexity for all parameters, complexity profile is lower. Such changes could be interpreted so, that more expressed is pathology leading to hypotension reactions of organism, lower complexity organism represent. Difference between episode 1 (before ABP fall down) and 2 (during ABP fall down) as and in Table I is small. These results rise up question what factors can influence such different reactions influencing all regulatory processes in organism and their interactions. Factor number one here could be the significance of injury, but to evaluate it from clinical point of view in real situation is very difficult or almost not impossible.

We can conclude, that problem is not possible to solve at current time. So we have investigated how patients of different genders react to those ABP falls. In Fig. 5 are presented women reaction to ABP fall, in Fig. 6 are shown men reactions to $\mathrm{ABP}$ falls. Comparing those two figures we can see, that men and women have different type of regulation of studied processes - complexity profiles differs. Due to small numbers of participants we can't make statistically significant conclusions, but we can postulate, from our data, that women are more stable in ABP fall episodes. Their complexity profile even slightly increases during ABP fall (Fig. 6), at the same situation men shows decrease of complexity profile during ABP fall (Fig. 7). So if we have situation with risk of ABP fall, for men it could be evaluated as more dangerous in comparison to women.

Such ABP fluctuation analysis methodology has a deep physical meaning. The bouts of quasistationarity; the evolution of the processes governed by a fixed algebraic law in each reconstructed segment can be identified. The proposed algorithm does not apply formal algebraic relationships for the observed data. It reveals that the hidden structure of the time series is able to identify potential changes in the evolution of the process and exploits predictability as a tool for the characterization of complexity [17].

\section{Profile of ECG parameters for group with hypotension reactions(Hy) and without}

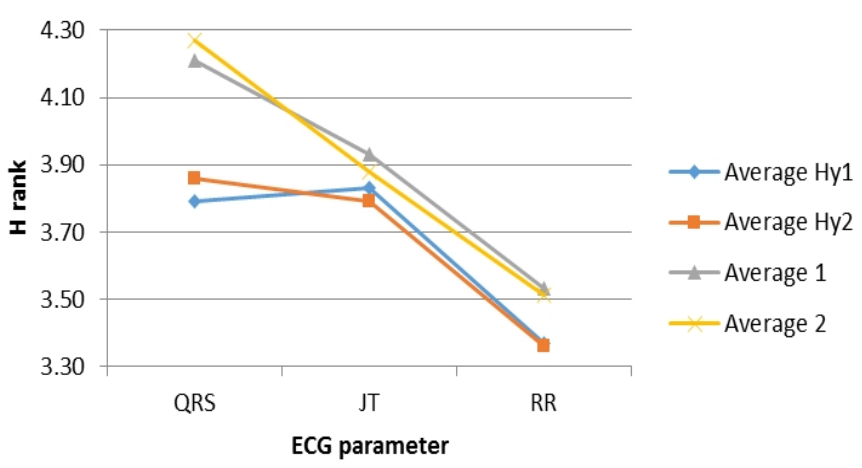

Fig. 4. The complexity profile of measured ECG parameters for persons with hypotension episodes (average Hy1 - for group $(n=24)$ of patients before hypotension episode and average Hy2 during hypotension) and without $(n=18)$ (average 1 before falling down $A B P$ and average 2 during decrease of $\mathrm{ABP}$, but not reaching hypotension level).

\section{Profile of ECG parameters for women with possible hypotension reactions}

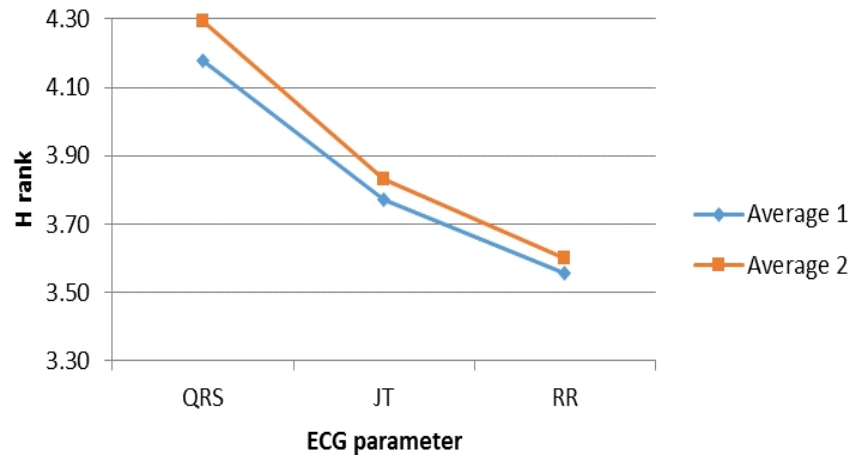

Fig. 5. The complexity profile of measured ECG parameters for women $(n=14)$. Average 1 line represent episode before ABP fall, average 2 line represent episode during $\mathrm{ABP}$ fall.
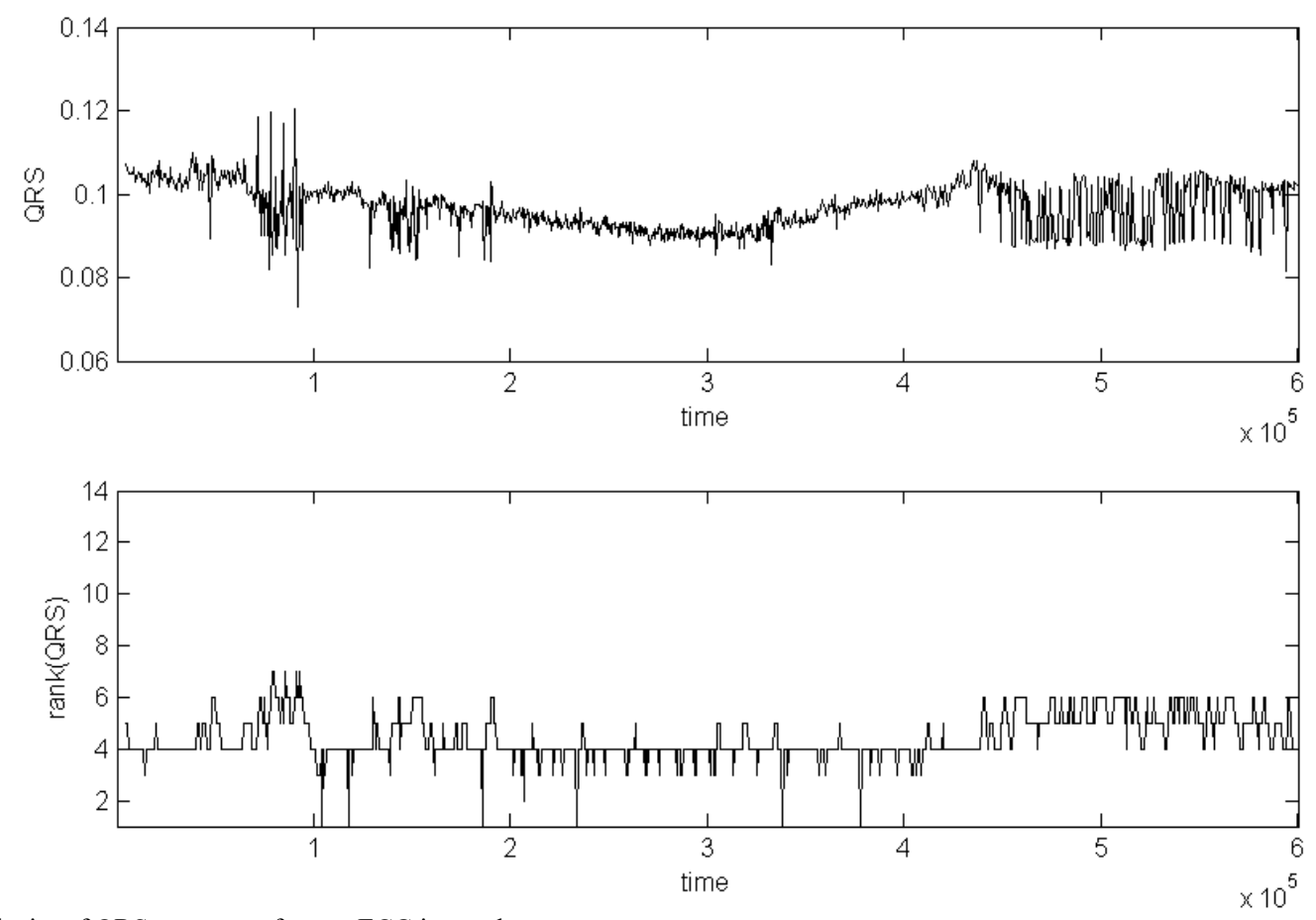

Fig. 6. The complexity of QRS parameter for one ECG interval. 
Profile of ECG parameters for men with possible hypotension reactions

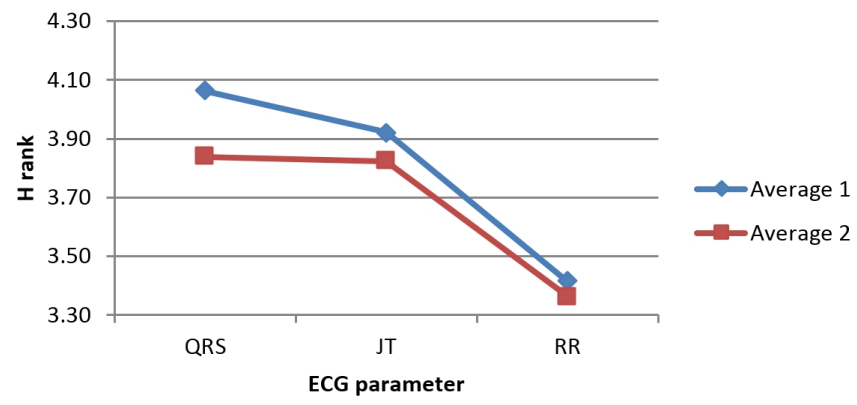

Fig. 7. The complexity profile of measured ECG parameters for men $(\mathrm{n}=$ 28). Average 1 line represent episode before ABP fall, average 2 line represent episode during ABP fall.

The application of the method for a time series analysis based on the identification of features of algebraic sequences is proposed in this paper. It can be noted that this method is especially effective when the time series are short. There are not always sufficient data to train the models, therefore such an approach when the features of algebraic sequence is identified from a short time series helps to extract as much information about the model of the process as possible and then use this model to extrapolate past behaviour into future.

\section{CONCLUSIONS}

Experimental results show that presented in this paper method also can be used together with other hypotension prediction methods. Hopefully proposed method could be useful in clinical patent after brain injuries monitoring and understanding of hypotension physiology. With the increasing amount of studies in this area and application of complex system theory into medicine it is hope to have more detailed and motivated interpretation of analysed data and data complexity itself.

\section{REFERENCES}

[1] A. Stell, R. Sinnott, R. Donald, I. Chambers et al, "Supporting clinical trials to predict adverse events in the brain trauma domain", 25th Int. Symposium on Computer-Based Medical Systems (CBMS), 2012, pp. 1-6. [Online]. Available: http://dx.doi.org/10.1109/cbms. 2012.6266380

[2] M. S. Pincus, "Approximate entropy as a measure of system complexity”, in Proc. Natl. Acad. Sci., 1991, vol. 88, pp. 2297-2301. [Online]. Available: http://dx.doi.org/10.1073/pnas.88.6.2297

[3] M. S. Pincus, "Assessing serial irregularity and its implication for health", Annals of the New York Academy of Sciences, vol. 954, no. 1, pp. 245-267, 2006. [Online]. Available: http://dx.doi.org/ 10.1111/j.1749-6632.2001.tb02755.x

[4] M. Costa, I. Cygankiewicz, W. Zareba, A. B. De Luna, A. L. Goldberger, S. Lobodzinski, "Multiscale complexity analysis of heart rate dynamics in heart failure: Preliminary findings from the music study", Computers in Cardiology, pp. 101-103, 2006.

[5] V. Yeragani, S. Appaya, K. Seema, R. Kumar, M. Tancer, "QRS amplitude of ECG in normal humans: effects of orthostatic challenge on linear and nonlinear measures of beat-to-beat variability", Cardiovascular Engineering: An International Journal, vol. 5, no. 3, pp. 135-140, 2005. [Online]. Available: http://dx.doi.org/10.1007/ s10558-005-7674-0

[6] A. Sliupaite, Z. Navickas, A. Vainoras, "Evaluation of complexity of ECG parameters using sample entropy and Hankel matrix", Elektronika ir Elektrotechnika, vol. 4, no. 92, pp. 107-110, 2009.

[7] E. D. Ubeyli, "Detecting variabilities of ECG signals by Lyapunov exponents", Neural Computing and Applications, vol. 18, no. 7, pp. 653-662, 2009. [Online]. Available: http://dx.doi.org/10.1007/ s00521-008-0229-8

[8] A. Cohen, "Hidden Markov models in biomedical signal processing", Paper presented at the 20th Annual Int. Conf. IEEE, Engineering in Medicine and Biology Society 3, pp. 1145-1150, 2009.

[9] S. Zhou, Z. Zhang, J. Gu, "Interpretation of coarse-graining of Lempel-Ziv complexity measure in ECG signal analysis", in Proc. Conf. IEEE Eng. Med. Biol. Soc., 2011, pp. 2716-2719.

[10] Z. Navickas, L. Bikulciene, "Expressions of solutions of ordinary differential equations by standard functions", Mathematical Modeling and Analysis, vol. 11, pp. 399-412, 2006.

[11] Z. Navickas, L. Bikulciene, M. Ragulskis, "Generalization of Expfunction and other standard function based methods", Applied Mathematics and Computations, vol. 216, no. 8, pp. 2380-2393, 2010. [Online]. Available: http://dx.doi.org/10.1016/j.amc.2010. 03.083

[12] Z. Navickas, M. Ragulskis, L. Bikulciene, "Special solutions of Huxley differential equation", Mathematical Modelling and Analysis, vol. 16, no. 2, pp. 248-259, 2011. [Online]. Available: http://dx.doi.org/10.3846/13926292.2011.579627

[13] M. Ragulskis, Z. Navickas, L. Bikulciene, "The solitary solution of the Liouville equation produced by the Exp-function method holds not for all initial conditions", Computers and Mathematics with Applications, vol. 62, pp. 367-382, 2011. [Online]. Available: http://dx.doi.org/10.1016/j.camwa.2011.05.020

[14] M. Ragulskis, K. Lukoseviciute, Z. Navickas, R. Palivonaite, "Shortterm time series forecasting based on the identification of skeleton algebraic sequences", Neurocomputing, vol. 64, no. 10, pp. 17351747, 2011. [Online]. Available: http://dx.doi.org/10.1016/j.neucom. 2011.02.017

[15] D. Karaliene, Z. Navickas, A. Slapsinskaite, A. Vainoras, "Investigation of the stability of fluctuations in electrocardiography data", Journal of Vibroengineering, vol. 15, no. 1, pp. 367-378, 2012.

[16] Y. Bar-Yam, "Complexity rising: From human beings to human civilization, a complexity profile", Encyclopedia of Life Support Systems (EOLSSUNESCO Publishers, Oxford, UK, 2002); also NECSI Report 1997-12-01, 1997.

[17] G. Boffetta, M. Cencini, F. Falcioni, A. Vulpiani, "Predictability: a way to characterize complexity", Phys. Rep., vol. 356, pp. 367-474, 2002. [Online]. Available: http://dx.doi.org/10.1016/S03701573(01)00025-4. 